\title{
On the period of the continued fraction for values of the square root of power sums
}

\author{
by \\ Amedeo Scremin (Udine)
}

1. Introduction. It is well known that the continued fraction for a rational number is finite and that the one for the square root of a positive integer $a$ which is not a square is periodic of the form $\left[a_{0} ; \overline{a_{1}, \ldots, a_{R-1}, 2 a_{0}}\right]$ (here $\overline{a_{1}, \ldots, a_{R-1}, 2 a_{0}}$ denotes the periodic part), where $R \geq 1$ is the length of the period. About $R$, we know that $R \ll \sqrt{a} \log a$ (see [4] and [6]).

A power sum $\alpha$ is a function on $\mathbb{N}$ of the form

$$
\alpha(n)=b_{1} c_{1}^{n}+b_{2} c_{2}^{n}+\cdots+b_{h} c_{h}^{n},
$$

where the roots $c_{i}$ are distinct integers and the coefficients $b_{i}$ are nonzero integers or rationals. We know from Corollary 1 in [2] that, apart from the case when $\alpha$ is the square of a power sum of the same kind on an arithmetic progression of naturals, $\sqrt{\alpha(n)}$ is a quadratic irrational for all but finitely many $n \in \mathbb{N}$. This means that the continued fraction expansion for $\sqrt{\alpha(n)}$ is periodic for $n$ large, raising the problem whether the length of the period is bounded or not for $n \rightarrow \infty$, which will be considered in this paper. This problem first appeared in the Final Remark (b) in [3], where it was predicted that "under suitable assumptions on the power sum $\alpha$ with rational roots and coefficients, the length of the period of the continued fraction for $\sqrt{\alpha(n)}$ tends to infinity with $n$ ". Very recently, Bugeaud and Luca (see [1]) found some partial results on the problem, giving a sufficient condition on $\alpha$, similar to that appearing in Corollary 3.3 below, under which the length of the period tends to infinity with $n$.

Remarkable results on a similar problem, but considering a nonconstant polynomial $f$ with rational coefficients instead of the power sum $\alpha$, were obtained by Schinzel in [7] and [8]. He provided necessary and sufficient

2000 Mathematics Subject Classification: 11J70, 11J25.

The author was supported by Istituto Nazionale di Alta Matematica "Francesco Severi", grant for abroad Ph.D. 
conditions on $f$ under which the length of the period of the continued fraction for $\sqrt{f(n)}$ tends to infinity as $n \rightarrow \infty$.

In the present paper we first prove that if a power sum $\alpha$ with rational coefficients cannot be approximated "too well" by the square of a power sum of the same kind (which implies that $\sqrt{\alpha(n)} \notin \mathbb{Q}$, i.e. its continued fraction is periodic, for all but finitely many $n \in \mathbb{N}$ ), then the length of the period of the continued fraction for $\sqrt{\alpha(n)}$ tends to infinity as $n \rightarrow \infty$ (Corollary 3.3).

Then we show that for any fixed $r \in\{0,1\}$, if the length of the period of the continued fraction for $\sqrt{\alpha(2 m+r)}$ is constant for all $m$ in an infinite set, then for all but finitely many $m$ in an arithmetic progression, the values of the partial quotients of the numerical continued fraction for $\sqrt{\alpha(2 m+r)}$ can be expressed by power sums of the same kind (Main Theorem 3.4). Moreover, we will prove that $\sqrt{\alpha(2 m+r)}$ has an identical continued fraction expansion involving power sums.

The results above will be deduced from some lower bounds for the quantities $|\sqrt{\alpha(n)}-p / q|$ (Corollary 3.2) and $|(\sqrt{\alpha(n)}+\beta(n)) / \gamma(n)-p / q|$ (Theorem 3.1) respectively, where $\alpha, \beta, \gamma$ are power sums and $p, q$ are integers, which we shall obtain using Schmidt's subspace theorem in a way similar to that of Corvaja and Zannier in [2] and [3].

Theorem 3.1 and Corollary 3.2 (for $\alpha=0$ and $q=1$ respectively) are the analogues of the Theorem in [3] and of Theorem 3 in [2].

The work in this paper carries out the suggestions in Final Remark (b) in [3].

2. Notation. In the present paper we will denote by $\Sigma$ the ring of functions on $\mathbb{N}$, called power sums, of the form

$$
\alpha(n)=b_{1} c_{1}^{n}+b_{2} c_{2}^{n}+\cdots+b_{h} c_{h}^{n},
$$

where the distinct roots $c_{i} \neq 0$, and the coefficients $b_{i} \neq 0$ are in $\mathbb{Z}$. For rings $A, B \subseteq \mathbb{C}$, let $A \Sigma_{B}$ denote the ring of power sums with coefficients in $A$ and roots in $B$. In the case $B=\mathbb{Z}$, we will write for simplicity $A \Sigma$ instead of $A \Sigma_{\mathbb{Z}}$.

If $B \subseteq \mathbb{R}$, it is enough to deal with power sums with only positive roots, since the positivity of the roots may be achieved by writing $2 n+r$ instead of $n$, and considering the cases of $r=0,1$ separately.

If $\alpha \in \overline{\mathbb{Q}} \Sigma_{\mathbb{Q}}$ is defined by $(2)$, we set $l(\alpha):=\max \left\{c_{1}, \ldots, c_{h}\right\}$. It is immediate that $l(\alpha \beta)=l(\alpha) l(\beta), l(\alpha+\beta) \leq \max \{l(\alpha), l(\beta)\}$ and $l(\alpha)^{n} \gg$ $|\alpha(n)| \gg l(\alpha)^{n}$.

Note. In the statements and proofs of our results we will always omit the condition of the existence of $\sqrt{\alpha(n)} \in \mathbb{R}$, i.e. that $\alpha(n) \geq 0$ for $n$ large. 
3. Statements. Theorem 3.1 below states that for power sums $\alpha, \beta, \gamma$ $\in \mathbb{Q} \Sigma$, if $(\sqrt{\alpha}+\beta) / \gamma$ cannot be well approximated on the subsequence of even (or odd) numbers by a power sum in $\mathbb{Q} \Sigma$, then $(\sqrt{\alpha(n)}+\beta(n)) / \gamma(n)$ cannot be well approximated by rationals with exponentially bounded denominators, except for a finite number of even (odd) $n$. This Diophantine approximation result will be obtained using Schmidt's subspace theorem in a way similar to that of Corvaja and Zannier in [2] and [3]. Theorem 3.1 is the main tool we will use to prove the corollaries and the Main Theorem.

Theorem 3.1. Let $\alpha, \beta, \gamma \in \mathbb{Q} \Sigma, \gamma$ not identically zero, and fix $\varepsilon>0$ and $r \in\{0,1\}$. Suppose that there does not exist a power sum $\eta \in \mathbb{Q} \Sigma$ such that

$$
\left|\frac{\sqrt{\alpha(2 m+r)}+\beta(2 m+r)}{\gamma(2 m+r)}-\eta(m)\right| \ll e^{-m \varepsilon} .
$$

Then there exist $k=k(\alpha, \beta, \gamma)>2$ and $Q=Q(\varepsilon)>1$ with the following properties. For all but finitely many naturals $n \equiv r \bmod 2$ and for all integers $p, q, 0<q<Q^{2 m+r}$, we have

$$
\left|\frac{\sqrt{\alpha(n)}+\beta(n)}{\gamma(n)}-\frac{p}{q}\right| \geq \frac{1}{q^{k}} e^{-\varepsilon n} .
$$

REMARK 1. Taking $\alpha=0$ in Theorem 3.1, we obtain again the result of the Theorem in [3].

Corollary 3.2 below is just a simplified version of Theorem 3.1, but we state it here because it is sufficient to prove Corollary 3.3. It states that if a power sum $\alpha \in \mathbb{Q} \Sigma$ cannot be well approximated on the subsequences of even and odd numbers by the square of a power sum from the same ring, then $\sqrt{\alpha(n)}$ cannot be well approximated by rationals with exponentially bounded denominators, except for a finite number of $n$.

To simplify the notation, we define

$$
\alpha_{r}(m):=\alpha(2 m+r) .
$$

Corollary 3.2. Let $\alpha \in \mathbb{Q} \Sigma$, and fix $\varepsilon>0$. Assume that for every $r \in\{0,1\}$ and for all $\xi \in \mathbb{Q} \Sigma$,

$$
l\left(\alpha_{r}-\xi^{2}\right) \geq l\left(\alpha_{r}\right)^{1 / 2}
$$

Then there exist $k=k(\alpha)>2$ and $Q=Q(\varepsilon)>1$ with the following property. For all but finitely many $n \in \mathbb{N}$ and for all integers $p, q, 0<q<Q^{n}$, we have

$$
\left|\sqrt{\alpha(n)}-\frac{p}{q}\right| \geq \frac{1}{q^{k}} e^{-\varepsilon n}
$$

Remark 2. Taking $q=1$, we can see that Corollary 3.2 is a generalization of Theorem 3 in [2]. 
REMARK 3. In concrete cases, it is easy to verify whether the assumption of Corollary 3.2 holds or not. Once $\alpha$ is given, it is enough to check with elementary algebraic methods, taking $r=0,1$ separately, that for all $\xi \in$ $\mathbb{Q} \Sigma$, in the power sum $\alpha_{r}-\xi^{2}$ there cannot be cancellations of all the coefficients of the roots with absolute value $\geq \sqrt{l\left(\alpha_{r}\right)}$. To do this, it is enough to check the cancellations for power sums $\xi$ with $l(\xi)=\sqrt{l\left(\alpha_{r}\right)}$, since otherwise $l\left(\alpha_{r}-\xi^{2}\right) \geq l\left(\alpha_{r}\right)^{1 / 2}$ holds automatically. Having this bound on the size $l$ of the dominant root of $\xi$, since $\xi \in \mathbb{Q} \Sigma$, the problem is reduced to a problem in a vector space of finite dimension, which can be easily handled with a system of algebraic equations.

By the same method, it is also easy to verify the assumption of Theorem 3.1.

The following Corollary 3.3 states that if a power sum $\alpha \in \mathbb{Q} \Sigma$ cannot be well approximated by the square of a power sum of the same kind, then the length of the period of the continued fraction for $\sqrt{\alpha(n)}$ tends to infinity as $n \rightarrow \infty$. This result was already obtained with a similar proof by Bugeaud and Luca in [1, Theorem 2.1].

Corollary 3.3. Let $\alpha \in \mathbb{Q} \Sigma$ be as in Corollary 3.2. Then the length of the period of the continued fraction for $\sqrt{\alpha(n)}$ tends to infinity as $n \rightarrow \infty$.

REMARK 4. Recall from the introduction that the assumption of Corollary 3.3 ensures that $\sqrt{\alpha(n)} \notin \mathbb{Q}$ for all but finitely many $n \in \mathbb{N}$, i.e. the period of the continued fraction for $\sqrt{\alpha(n)}$ is well defined for all $n$ large enough.

The Main Theorem 3.4 below follows again from Theorem 3.1, and states that if the length of the period of the continued fraction for the square root of a power sum is constant for infinitely many even (resp. odd) $n$, then the values of the partial quotients of the numerical continued fraction can be expressed by power sums on an arithmetic progression of even (resp. odd) $n$, except finitely many.

We will say that the functional relation

$$
\sqrt{\alpha}=\left[\beta_{0} ; \overline{\beta_{1}, \ldots, \beta_{R}}\right]
$$

holds identically if, putting $\tau:=\left[\overline{\beta_{1}, \ldots, \beta_{R}}\right]$, the second degree algebraic relation

$$
\alpha \tau^{2}=\left(\beta_{0} \tau+1\right)^{2}
$$

holds in the ring of power sums.

Main Theorem 3.4. Let $\alpha \in \mathbb{Q} \Sigma$, and fix $r \in\{0,1\}$. Suppose that there exists an infinite set $A \subseteq \mathbb{N}$ and a constant $R \geq 0$ such that for $m \in A$ the length of the period of the continued fraction expansion for $\sqrt{\alpha(2 m+r)}$ is $R$. Then there exist an arithmetic progression $\mathcal{P}$ containing infinitely many 
elements of $A$, and power sums $\beta_{0}, \ldots, \beta_{R} \in \mathbb{Q} \Sigma$ integer-valued on the progression $\mathcal{P}$, such that for all but finitely many $m \in \mathcal{P}$, we have the numerical continued fraction expansion

$$
\sqrt{\alpha(2 m+r)}=\left[\beta_{0}(m) ; \overline{\beta_{1}(m), \ldots, \beta_{R}(m)}\right] .
$$

Moreover, the functional continued fraction expansion (5) holds identically.

Remark 5. The case $R=0$ of the Main Theorem states that for $\alpha \in$ $\mathbb{Q} \Sigma$, if $\sqrt{\alpha(2 m+r)} \in \mathbb{Z}$ for infinitely many $m \in \mathbb{N}$, then $\alpha(2 m+r)$ is the square of a power sum in $\mathbb{Q} \Sigma$. This is a particular case of Corollary 1 in [2].

REMARK 6. The result of Corollary 3.3, together with the Main Theorem 3.4, carries out the program outlined in the Final Remark (b) in [3].

REMARK 7. Under the assumption of the Main Theorem, it remains an open problem whether the length of the continued fraction for $\sqrt{\alpha(2 m+r)}$ is uniformly bounded for all $m \in \mathbb{N}$. For the polynomial case studied by Schinzel [8], he proved that even when the numerical continued fraction for $\sqrt{f(n)}$, where $f \in \mathbb{Z}[x]$, is bounded for infinitely many $n$ and $\sqrt{f(n)}$ admits a functional continued fraction expansion with bounded length (i.e. the same situation as above), the length of the numerical continued fraction expansion can tend to infinity on some subset $E \subseteq \mathbb{N}$. Schinzel provided a full characterization of such sets $E$ (Theorems 2 and 3 in [8]).

4. Auxiliary results. We state a version of Schmidt's subspace theorem due to H. P. Schlickewei, which will be our main tool to prove Theorem 3.1. It can be found in [10, Theorem 1E, p. 178] (a complete proof requires also [9]).

THEOREM 4.1. Let $S$ be a finite set of absolute values of $\mathbb{Q}$, including the infinite one and normalized in the usual way (i.e. $|p|_{v}=p^{-1}$ if $\left.v \mid p\right)$. Extend each $v \in S$ to $\overline{\mathbb{Q}}$ in some way. For $v \in S$ let $L_{1, v}, \ldots, L_{n, v}$ be $n$ linearly independent linear forms in $n$ variables with algebraic coefficients and let $\delta>0$. Then the solutions $\underline{x}:=\left(x_{1}, \ldots, x_{n}\right) \in \mathbb{Z}^{n}$ to the inequality

$$
\prod_{v \in S} \prod_{i=1}^{n}\left|L_{i, v}(\underline{x})\right|_{v}<\max _{1 \leq i \leq n}\left|x_{i}\right|^{-\delta}
$$

are contained in finitely many proper subspaces of $\mathbb{Q}^{n}$.

The following lemma is a special case of a result by Evertse; a short proof can be found in [2, Lemma 2].

Lemma 4.2. Let $\xi \in \mathbb{Q} \Sigma_{\mathbb{Q}}$ and let $D$ be the minimal positive integer such that $D^{n} \xi \in \mathbb{Q} \Sigma$. Then, for every $\varepsilon>0$, there are only finitely many $n \in \mathbb{N}$ such that the denominator of $\xi(n)$ is smaller than $D^{n} e^{-n \varepsilon}$. 
5. Proofs. We start with the following very simple

Lemma 5.1. Let $\alpha, \beta, \gamma \in \mathbb{Q} \Sigma, \gamma$ not identically zero, and let $t$ be any positive real number. Then for every $r \in\{0,1\}$ there exists $\eta_{r} \in \overline{\mathbb{Q}} \Sigma_{\mathbb{Q}}$ such that

$$
\left|\frac{\sqrt{\alpha(2 m+r)}+\beta(2 m+r)}{\gamma(2 m+r)}-\eta_{r}(2 m+r)\right| \ll t^{2 m} \text {. }
$$

Such an $\eta_{r}$ can be effectively computed in terms of $r, \alpha, \beta, \gamma$ and $t$.

Proof. Let $\alpha(n)=\sum_{j=1}^{h} b_{j} c_{j}^{n}$ with $c_{j} \in \mathbb{Z}, c_{j} \neq 0$ and $b_{j} \in \mathbb{Q}^{*}$ for all $j=1, \ldots, h$. We can suppose $c_{1}>\cdots>c_{h}>0$. For a real (resp. real positive) determination of $b_{1}^{1 / 2}$ (resp. $c_{1}^{1 / 2}$ ), fixed for the rest of the proof, we have

$$
\alpha(n)^{1 / 2}=\left(b_{1} c_{1}^{n}\right)^{1 / 2}\left(1+\sum_{j=2}^{h} \frac{b_{j}}{b_{1}}\left(\frac{c_{j}}{c_{1}}\right)^{n}\right)^{1 / 2}=\left(b_{1} c_{1}^{n}\right)^{1 / 2}(1+\sigma(n))^{1 / 2}
$$

with $\sigma(n) \in \mathbb{Q} \Sigma_{\mathbb{Q}}$, and $\sigma(n)=O\left(\left(c_{2} / c_{1}\right)^{n}\right)$.

Expanding the function $x \mapsto(1+x)^{1 / 2}$ in a Taylor series, we have

$$
(1+\sigma(n))^{1 / 2}=1+\sum_{j=1}^{H}\left(\begin{array}{c}
1 / 2 \\
j
\end{array}\right) \sigma(n)^{j}+O\left(|\sigma(n)|^{H+1}\right),
$$

where $H>0$ is an integer that can be chosen later. For every $r \in\{0,1\}$, substituting (7) in (6) we obtain

$$
\begin{aligned}
\alpha(2 m+r)^{1 / 2}= & b_{1}^{1 / 2} c_{1}^{r / 2} c_{1}^{m}\left(1+\sum_{j=1}^{H}\left(\begin{array}{c}
1 / 2 \\
j
\end{array}\right) \sigma(2 m+r)^{j}\right) \\
& +O\left(\left(\frac{c_{2}}{c_{1}}\right)^{2 m(H+1)} c_{1}^{m}\right) .
\end{aligned}
$$

Let

$$
\beta(n)=\sum_{j=1}^{k} d_{j} e_{j}^{n} \in \mathbb{Q} \Sigma
$$

with $e_{j} \in \mathbb{Z}, e_{j} \neq 0$ and $d_{j} \in \mathbb{Q}^{*}$ for all $j=1, \ldots, k$. We can suppose $e_{1}>\cdots>e_{k}>0$. Fix $H$ such that $\left(c_{2} / c_{1}\right)^{H+1} c_{1}^{1 / 2}<e_{1}$.

Let $\gamma(n)=\sum_{j=1}^{l} f_{j} g_{j}^{n} \in \mathbb{Q} \Sigma$ with $g_{j} \in \mathbb{Z}, g_{j} \neq 0$ and $f_{j} \in \mathbb{Q}^{*}$ for all $j=1, \ldots, l$.

We can suppose $g_{1}>\cdots>g_{k}>0$. Using the same method as in the proof of Theorem 1 in [2], we can write

$$
\gamma(n)^{-1}=f_{1}^{-1} g_{1}^{-n} \sum_{j=0}^{s} \phi(n)^{j}+O\left(\left(g_{2} / g_{1}\right)^{n(s+1)} g_{1}^{-n}\right),
$$


where

$$
\phi(n):=-\sum_{i=2}^{l} \frac{f_{i}}{f_{1}}\left(\frac{g_{i}}{g_{1}}\right)^{n} \in \mathbb{Q} \Sigma_{\mathbb{Q}},
$$

$\phi(n)=O\left(g_{2} / g_{1}\right)^{n}$, and $s>0$ is an integer that will be chosen later.

Thus, by equations (8)-(10), by the choice of $H$ and the definition of $\phi$, we obtain

$$
\begin{gathered}
\frac{\sqrt{\alpha(2 m+r)}+\beta(2 m+r)}{\gamma(2 m+r)}=f_{1}^{-1} g_{1}^{-r} g_{1}^{-2 m}\left(\sum_{i=0}^{s} \phi(2 m+r)^{i}\right) \\
\times\left(b_{1}^{1 / 2} c_{1}^{r / 2} c_{1}^{m}\left(1+\sum_{i=1}^{H}\left(\begin{array}{c}
1 / 2 \\
j
\end{array}\right) \sigma(2 m+r)^{i}\right)+\sum_{i=1}^{k} d_{i} e_{i}^{2 m+r}\right) \\
+O\left(\left(g_{2} / g_{1}\right)^{2 m(s+1)} g_{1}^{-2 m} e_{1}^{2 m}\right) .
\end{gathered}
$$

Fix now $s$ such that $\left(g_{2} / g_{1}\right)^{s+1} g_{1}^{-1} e_{1}<t$ and put, for $r=0,1$,

$$
\begin{aligned}
\eta_{r}(2 m+r):= & f_{1}^{-1} g_{1}^{-r} g_{1}^{-2 m}\left(\sum_{i=0}^{s} \phi(2 m+r)^{i}\right) \\
& \times\left(b_{1}^{1 / 2} c_{1}^{r / 2} c_{1}^{m}\left(1+\sum_{i=1}^{H}\left(\begin{array}{c}
1 / 2 \\
j
\end{array}\right) \sigma(2 m+r)^{i}\right)+\sum_{i=1}^{k} d_{i} e_{i}^{2 m+r}\right) .
\end{aligned}
$$

By definition $\eta_{r} \in \overline{\mathbb{Q}} \Sigma_{\mathbb{Q}}$ for $r=0,1$. Thus for $r \in\{0,1\}$ we have effectively constructed a power sum $\eta_{r}(n) \in \overline{\mathbb{Q}} \Sigma_{\mathbb{Q}}$ such that

$$
\left|\frac{\sqrt{\alpha(2 m+r)}+\beta(2 m+r)}{\gamma(2 m+r)}-\eta_{r}(2 m+r)\right| \ll t^{2 m},
$$

completing the proof.

REMARK 8. Notice that in $\eta_{r}$ the root with largest absolute value is $g_{1}^{-1} \max \left\{e_{1}, c_{1}^{1 / 2}\right\}$ and that the other roots appearing are rational with denominator of the form $c_{1}^{a} g_{1}^{b}$ with $a, b \in \mathbb{N}, a \geq 0, b \geq 1$.

Proof of Theorem 3.1. Let $\eta_{r}$, for $r \in\{0,1\}$ fixed, be as in Lemma 5.1, with $t=1 / 9$. We can write (recall Remark 8 , and the definition of $g_{1}$ in the proof of Lemma 5.1)

$$
\eta_{r}(2 m+r)=b_{1, r}^{1 / 2} d_{1}^{m}\left(g_{1}^{-2 m}+b_{2} d_{2}^{2 m+r}+\cdots+b_{h} d_{h}^{2 m+r}\right)
$$

for some $b_{1, r}, b_{i} \in \overline{\mathbb{Q}}^{*}, d_{1}, g_{1} \in \mathbb{Z} \backslash\{0\}, d_{2}, \ldots, d_{h} \in \mathbb{Q}^{*}$, and $g_{1}^{-1}>d_{2}>$ $\cdots>d_{h}>0$.

We define $k:=h+3$ and, for $\varepsilon>0$ fixed (which we may take $<1 / 2 k$, say), $Q:=e^{\varepsilon}$. We suppose that there are infinitely many triples $(m, p, q)$ of 
integers with $0<q<Q^{2 m+r}, m \rightarrow \infty$ and

$$
\left|\frac{\sqrt{\alpha(2 m+r)}+\beta(2 m+r)}{\gamma(2 m+r)}-\frac{p}{q}\right| \leq \frac{1}{q^{k}} e^{-\varepsilon(2 m+r)} .
$$

We shall eventually obtain a contradiction, which will prove what we want.

We proceed to define the data for an application of the Subspace Theorem 4.1. We let $S$ be the finite set of places of $\mathbb{Q}$ containing the infinite one and all the places dividing the numerators or denominators of $g_{1}$ and of $d_{i}, i=1, \ldots, h$. We define linear forms on $X_{0}, \ldots, X_{h}$ as follows. If $v \neq \infty$ or $i \neq 0$ we set simply $L_{i, v}=X_{i}$. We define the remaining form by

$$
L_{0, \infty}:=X_{0}-b_{1, r}^{1 / 2} X_{1}-b_{2, r} X_{2}-\cdots-b_{h, r} X_{h},
$$

where $b_{i, r}=b_{i} b_{1, r}^{1 / 2}, i=2, \ldots, h$. For each $v$, these linear forms are clearly independent.

Let $d$ be the minimal integer such that $d_{i} d \in \mathbb{Z}$ for every $i=1, \ldots, h$ (recall Remark 8). For our choice of the set $S, d$ is an $S$-unit.

Define $e_{1}:=d_{1} d g_{1}^{-2}, e_{i}:=d d_{i}, i=2, \ldots, h$. Note that $e_{i} \in \mathbb{Z}$ for every $i=1, \ldots, h$. Set

$$
\underline{x}=\underline{x}(m, p, q)=\left(p d^{2 m+r}, q e_{1}^{m} d^{m+r}, q d_{1}^{m} e_{2}^{2 m+r}, \ldots, q d_{1}^{m} e_{h}^{2 m+r}\right) \in \mathbb{Z}^{h+1} .
$$

We now estimate the double product $\prod_{v \in S} \prod_{i=0}^{h}\left|L_{i, v}(\underline{x})\right|_{v}$. We have

$$
\prod_{v \in S} \prod_{i=0}^{h}\left|L_{i, v}(\underline{x})\right|_{v}=\left|L_{0, \infty}(\underline{x})\right| \cdot \prod_{i=1}^{h} \prod_{v \in S}\left|L_{i, v}(\underline{x})\right|_{v} \cdot \prod_{v \in S \backslash\{\infty\}}\left|L_{0, v}(\underline{x})\right|_{v} .
$$

By definition $\prod_{v \in S}\left|L_{1, v}(\underline{x})\right|_{v}=\prod_{v \in S}\left|q e_{1}^{m} d^{m+r}\right|_{v} \leq q$ and, for $i \geq 2$, $\prod_{v \in S}\left|L_{i, v}(\underline{x})\right|_{v}=\prod_{v \in S}\left|q d_{1}^{m} e_{i}^{2 m+r}\right|_{v} \leq q$, since $d, d_{1}$ and the $e_{i}$ are $S$-units for every $i$ (which implies that $\prod_{v \in S}|d|_{v}=\prod_{v \in S}\left|d_{1}\right|_{v}=\prod_{v \in S}\left|e_{i}\right|_{v}=1$ ) and since $\prod_{v \in S}|q|_{v} \leq q$ for the positive integer $q$. This means that

$$
\prod_{i=1}^{h} \prod_{v \in S}\left|L_{i, v}(\underline{x})\right|_{v} \leq q^{h}
$$

Moreover,

$$
\begin{aligned}
\prod_{v \in S \backslash\{\infty\}}\left|L_{0, v}(\underline{x})\right|_{v} & =\prod_{v \in S \backslash\{\infty\}}\left|p d^{2 m+r}\right|_{v} \\
& =\prod_{v \in S \backslash\{\infty\}}|p|_{v} \cdot \prod_{v \in S \backslash\{\infty\}}\left|d^{2 m+r}\right|_{v} \leq d^{-(2 m+r)},
\end{aligned}
$$

the last inequality holding since $p$ is an integer and $d$ is an $S$-unit. 
Finally, we have

$$
\begin{aligned}
\left|L_{0, \infty}(\underline{x})\right| & =d^{2 m+r}\left|p-q\left(b_{1, r}^{1 / 2} d_{1}^{m} g_{1}^{-2 m}+b_{2, r} d_{1}^{m} d_{2}^{2 m+r}+\cdots+b_{h, r} d_{1}^{m} d_{h}^{2 m+r}\right)\right| \\
& =q d^{2 m+r}\left|\eta_{r}(2 m+r)-p / q\right|,
\end{aligned}
$$

which, combined with (12)-(14), gives

$$
\prod_{v \in S} \prod_{i=0}^{h}\left|L_{0, v}(\underline{x})\right|_{v} \leq q^{h+1}\left|\eta_{r}(2 m+r)-p / q\right| .
$$

Since $q^{k}<Q^{k(2 m+r)}=e^{(2 m+r) k \varepsilon}$, we have $q^{-k} e^{-(2 m+r) \varepsilon}>e^{-(2 m+r)(k+1) \varepsilon}$, which means that $q^{-k} e^{-(2 m+r) \varepsilon}>t^{2 m+r}$ (recall that $\varepsilon<1 / 2 k, k \geq 3$ and $t=1 / 9)$. Thus, for a certain constant $l>0$, we have

$$
\begin{aligned}
\left|\eta_{r}(2 m+r)-\frac{p}{q}\right| \leq & \left(\left|\frac{p}{q}-\frac{\sqrt{\alpha(2 m+r)}+\beta(2 m+r)}{\gamma(2 m+r)}\right|\right. \\
& \left.+\left|\frac{\sqrt{\alpha(2 m+r)}+\beta(2 m+r)}{\gamma(2 m+r)}-\eta_{r}(2 m+r)\right|\right) \\
\leq & \left(\frac{1}{q^{k}} e^{-(2 m+r) \varepsilon}+l t^{2 m+r}\right) \leq \frac{2}{q^{k}} e^{-(2 m+r) \varepsilon} .
\end{aligned}
$$

This means that

$$
\prod_{v \in S} \prod_{i=0}^{h}\left|L_{0, v}(\underline{x})\right|_{v} \leq 2 q^{h+1-k} e^{-(2 m+r) \varepsilon} \leq e^{-(2 m+r) \varepsilon},
$$

since we have $k=h+3$. Also,

$$
\max _{0 \leq i \leq h}\left|x_{i}\right| \simeq q e_{1}^{m} d^{m+r} \leq Q^{2 m+r} e_{1}^{m} d^{m+r} .
$$

Hence, choosing $\delta>0$ with $\delta<\varepsilon / \log \left(Q^{2} e_{1} d\right)$, we get, for $m$ large,

$$
\prod_{v \in S} \prod_{i=0}^{h}\left|L_{0, v}(\underline{x})\right|_{v} \leq e^{-(2 m+r) \varepsilon}<\left(Q^{2 m+r} e_{1}^{m} d^{m+r}\right)^{-\delta} \leq\left(\max _{0 \leq i \leq h}\left|x_{i}\right|\right)^{-\delta},
$$

i.e. the inequality of the Subspace Theorem 4.1 is satisfied. This implies that the vectors

$$
\underline{x}=\underline{x}(m, p, q)=\left(p d^{2 m+r}, q e_{1}^{m} d^{m+r}, q d_{1}^{m} e_{2}^{2 m+r}, \ldots, q d_{1}^{m} e_{h}^{2 m+r}\right) \in \mathbb{Z}^{h+1}
$$

are contained in a finite set of proper subspaces of $\mathbb{Q}^{h+1}$. In particular, there exists a fixed subspace, say of equation $z_{0} X_{0}-z_{1} X_{1}-\cdots-z_{h} X_{h}=0, z_{i} \in \mathbb{Q}$, containing infinitely many of the vectors in question. We cannot have $z_{0}=0$, since this would entail

$$
\begin{aligned}
z_{1} e_{1}^{m} d^{m+r}+z_{2} d_{1}^{m} & e_{2}^{2 m+r}+\cdots+z_{h} d_{1}^{m} e_{h}^{2 m+r} \\
& =d_{1}^{m} d^{2 m+r}\left(z_{1} g_{1}^{-2 m}+z_{2} d_{2}^{2 m+r}+\cdots+z_{h} d_{h}^{2 m+r}\right)=0
\end{aligned}
$$


for infinitely many $m$; in turn, the fact that $g^{-1}$ and the $d_{i}$ are pairwise distinct would imply $z_{i}=0$ for all $i$, a contradiction.

Therefore we can suppose that $z_{0}=1$, and we find that, for $m$ corresponding to the vectors in question,

$$
\frac{p}{q}=d_{1}^{m}\left(z_{1} g_{1}^{-2 m}+\sum_{i=2}^{h} z_{i} d_{i}^{2 m+r}\right)=: \xi(m) \in \mathbb{Q} \Sigma_{\mathbb{Q}} .
$$

Let us show that actually $\xi \in \mathbb{Q} \Sigma$. Assume the contrary; then the minimal positive integer $D$ so that $D^{m} \xi \in \mathbb{Q} \Sigma$ is $\geq 2$. But then equation (16) together with Lemma 4.2 implies that $q \gg 2^{m} e^{-m \varepsilon}$. Since this would hold for infinitely many $m$, we would find $Q \geq q^{1 / 2 m} \geq \sqrt{2} e^{-\varepsilon / 2}$, a contradiction since $Q=e^{\varepsilon}, \varepsilon<1 / 2 k$ and $k \geq 3$. Therefore $\xi \in \mathbb{Q} \Sigma$.

Substituting (16) in (11) we find that there exists a power sum $\xi \in \mathbb{Q} \Sigma$ such that

$$
\left|\frac{\sqrt{\alpha(2 m+r)}+\beta(2 m+r)}{\gamma(2 m+r)}-\xi(m)\right| \ll e^{-2 m \varepsilon},
$$

a contradiction, concluding the proof.

Proof of Corollary 3.2. We know that

$$
l\left(\alpha_{r}-\xi^{2}\right) \geq l\left(\alpha_{r}\right)^{1 / 2}
$$

for every $\xi \in \mathbb{Q} \Sigma$ by assumption, and for every $r \in\{0,1\}$,

$$
\left|\sqrt{\alpha_{r}(m)}+\xi_{r}(m)\right|<2 \max \left\{\sqrt{\alpha_{r}(m)},\left|\xi_{r}(m)\right|\right\} .
$$

If for a certain $\xi \in \mathbb{Q} \Sigma$ we have $\left|\xi_{r}(m)\right| \leq k \sqrt{\alpha_{r}(m)}$ for $m$ large enough and for some constant $k>0$, then for such $\xi \in \mathbb{Q} \Sigma$ and $m$ large,

$$
\left|\sqrt{\alpha_{r}(m)}-\xi_{r}(m)\right|>\frac{1}{2} \min \left\{1, \frac{1}{k}\right\} .
$$

If for a certain $\xi \in \mathbb{Q} \Sigma$ we have $\left|\xi_{r}(m)\right| \gg \alpha_{r}(m)^{(1+\delta) / 2}$ for some $\delta>0$, we get

$$
\left|\sqrt{\alpha_{r}(m)}-\xi_{r}(m)\right| \gg \alpha_{r}(m)^{(1+\delta) / 2} .
$$

This proves that there does not exist a power sum $\xi \in \mathbb{Q} \Sigma$ and $\varepsilon>0$ such that

$$
\left|\sqrt{\alpha_{r}(m)}-\xi_{r}(m)\right| \ll e^{-2 m \varepsilon} .
$$

Thus we can apply Theorem 3.1 with $\beta=0$ and $\gamma=1$, and get the conclusion.

Proof of Corollary 3.3. For notation and basic facts about continued fractions we refer to [5] and [9, Ch. I].

Recall from Remark 4 that under our present assumption the period of the continued fraction for $\sqrt{\alpha(n)}$ is well defined for all $n$ large enough. 
Suppose by contradiction that there exists an integer $R>0$ and an infinite set $A \subseteq \mathbb{N}$ such that $\sqrt{\alpha(n)}=\left[a_{0}(n) ; \overline{a_{1}(n), \ldots, a_{R}(n)}\right]$ for $n \in A$. Let $p_{i}(n) / q_{i}(n), i=0,1, \ldots$, with $q_{0}(n)=1$, be the (infinite) sequence of convergents of the continued fraction for $\sqrt{\alpha(n)}$. We recall the relation

$$
\left|\sqrt{\alpha(n)}-\frac{p_{i}(n)}{q_{i}(n)}\right|<\left(a_{i+1}(n) q_{i}(n)^{2}\right)^{-1} \quad \text { for } i \geq 0,
$$

which implies that

$$
a_{i+1}(n)<\left|\sqrt{\alpha(n)}-\frac{p_{i}(n)}{q_{i}(n)}\right|^{-1} q_{i}(n)^{-2} \quad \text { for } i \geq 0 .
$$

Since $\alpha$ satisfies the assumptions of Corollary 3.2, for some $\varepsilon>0$ to be fixed later there exist $k>2$ and $Q>1$ as in the statement of Corollary 3.2. As in the proof of Theorem 3.1 (from which Corollary 3.2 follows), we can take $Q=e^{\varepsilon}$.

Define now the increasing sequence $c_{0}, c_{1}, \ldots$ by $c_{0}=0$ and $c_{r+1}=$ $(k+1) c_{r}+1$, and choose a positive number $\varrho<c_{R}^{-1} \log Q$, so $e^{c_{R} \varrho}<Q$. Proceeding by induction as in the proof of Corollary 1 in [3], it can be shown that for every $i=0, \ldots, R$, and for large $n$, we have $q_{i}(n)<e^{c_{i} \varrho n}$, which implies that $q_{i}(n)<Q^{n}$ for every $i=0, \ldots, R$ and $n$ large. Thus, we can apply Corollary 3.2 with $p=p_{i}(n), q=q_{i}(n)$, and $\varepsilon>0$ to be chosen later. Recalling that $Q=e^{\varepsilon}$, from (17) we see that, for all but finitely many $n$,

$$
\begin{aligned}
a_{i+1}(n) & <\left|\sqrt{\alpha(n)}-\frac{p_{i}(n)}{q_{i}(n)}\right|^{-1} q_{i}(n)^{-2} \leq q_{i}(n)^{k} e^{n \varepsilon} \\
& <Q^{k n} e^{n \varepsilon}=e^{n(k+1) \varepsilon}
\end{aligned}
$$

for every $i=0, \ldots, R$ and $\varepsilon>0$. Taking $\delta:=(k+1) \varepsilon$ we can rewrite the above inequality as

$$
a_{i}(n)<e^{n \delta}
$$

for $i=0, \ldots, R$ and for all but finitely many $n$.

From now on, let $n \in A$ be such that $a_{i}(n)<e^{n \delta}$. By assumption, for every $n$,

$$
\sqrt{\alpha(n)}=a_{0}(n)+\frac{1}{\beta(n)},
$$

where $\beta(n)$ has the continued fraction expansion

$$
\beta(n)=\left[\overline{a_{1}(n), \ldots, a_{R}(n)}\right] .
$$

This means that $\beta(n)$ satisfies

$$
\beta(n)=\left[a_{1}(n), \ldots, a_{R}(n), \beta(n)\right],
$$

which can be rewritten as a quadratic equation

$$
q_{R}^{\prime}(n) \beta(n)^{2}+\left(q_{R-1}^{\prime}(n)-p_{R}^{\prime}(n)\right) \beta(n)-p_{R-1}^{\prime}(n)=0,
$$


where $p_{i}^{\prime}(n) / q_{i}^{\prime}(n)=\left[a_{1}(n), \ldots, a_{i}(n)\right]$. It is well known that $p_{i}^{\prime}(n), q_{i}^{\prime}(n)$ satisfy the recursive equations $p_{i+2}^{\prime}(n)=a_{i+2}(n) p_{i+1}^{\prime}(n)+p_{i}^{\prime}(n)$ and $q_{i+2}^{\prime}(n)=$ $a_{i+2}(n) q_{i+1}^{\prime}(n)+q_{i}^{\prime}(n)$ for all $i \geq-1$, with initial values $p_{0}^{\prime}(n)=q_{-1}^{\prime}(n)=1$ and $q_{0}^{\prime}(n)=p_{-1}^{\prime}(n)=0$. It follows that the integers $p_{R-1}^{\prime}(n), p_{R}^{\prime}(n), q_{R-1}^{\prime}(n)$ and $q_{R}^{\prime}(n)$ appearing in $(21)$ are all $\ll\left(\max _{1 \leq i \leq R} a_{i}(n)\right)^{R}$.

From (19) it follows that $\max _{1 \leq i \leq R} a_{i}(n)<e^{n \delta}$, which implies that $p_{R-1}^{\prime}(n), p_{R}^{\prime}(n), q_{R-1}^{\prime}(n)$ and $q_{R}^{\prime}(n)$ are all $\ll e^{R n \delta}$. Taking the trace of both terms of (20) we see that for infinitely many $n$,

$$
2 a_{0}(n)=\frac{q_{R-1}^{\prime}(n)-p_{R}^{\prime}(n)}{p_{R-1}^{\prime}(n)} .
$$

Estimating the absolute value on both sides of (22), on the left side we get

$$
\left|2 a_{0}(n)\right|=2\lfloor\sqrt{\alpha(n)}\rfloor \gg 2^{n / 2}
$$

(since $\alpha$ can be supposed to be a nonconstant power sum), while on the right side we have

$$
\left|\frac{q_{R-1}^{\prime}(n)-p_{R}^{\prime}(n)}{p_{R-1}^{\prime}(n)}\right| \ll\left|q_{R-1}^{\prime}(n)\right|+\left|p_{R}^{\prime}(n)\right| \ll e^{R n \delta},
$$

yielding a contradiction for $\delta<(\ln 2) / 2 R$, i.e. $\varepsilon<(\ln 2) / 2(k+1) R$.

Proof of the Main Theorem 3.4. The case of $\alpha$ constant is trivial; thus we can suppose $\alpha$ to be nonconstant for the rest of the proof. For $r \in\{0,1\}$ fixed, let

$$
\sqrt{\alpha(2 m+r)}=\left[a_{0}(m) ; a_{1}(m), a_{2}(m), \ldots\right]=\left[a_{0}(m) ; \overline{a_{1}(m), \ldots, a_{R(m)}(m)}\right]
$$

be the numerical continued fraction expansion for $\sqrt{\alpha(2 m+r)}$, and let $p_{i}(m) / q_{i}(m), i=0,1, \ldots$, with $q_{0}(m)=1$, be the (infinite) sequence of its convergents. If $m \in A$, we have $R(m)=R$.

We recall the relations $a_{R}(m)=2 a_{0}(m)$ for every $m \in A$ (if $R>0$ ), and

$$
a_{i+1}(m)<\left|\sqrt{\alpha(2 m+r)}-\frac{p_{i}(m)}{q_{i}(m)}\right|^{-1} q_{i}(m)^{-2}
$$

for every $i \geq 0$ and $m \in \mathbb{N}$.

By our present assumption, the hypothesis of Corollary 3.3 cannot hold for $\alpha$ and for the fixed $r$, since the period of the continued fraction for $\sqrt{\alpha(n)}$ cannot tend to infinity as $n \rightarrow \infty$. This means that for some $\varrho>0$, there exists a power sum $\eta \in \mathbb{Q} \Sigma$ such that

$$
\left|\alpha(2 m+r)-\eta(m)^{2}\right| \ll \alpha(2 m+r)^{1 / 2-\varrho} .
$$

From (24) it follows that

$$
|\sqrt{\alpha(2 m+r)}-\eta(m)| \ll \alpha(2 m+r)^{-\varrho}<1,
$$


the last inequality holding for $m \in \mathbb{N}$ large. It follows that for every large enough $m \in \mathbb{N}$,

$$
a_{0}(m)=\lfloor\sqrt{\alpha(2 m+r)}\rfloor \in\{\lfloor\eta(m)\rfloor-1,\lfloor\eta(m)\rfloor,\lfloor\eta(m)\rfloor+1\} .
$$

Since $\eta$ has integral roots and rational coefficients, there exist arithmetic progressions $A_{s}=\left\{m=t m^{\prime}+s: m^{\prime} \in \mathbb{N}\right\}$, for $s=0, \ldots, t-1$ and some $t \in \mathbb{N}$, such that for all $m \in A_{s}$ we have $\lfloor\eta(m)\rfloor=\zeta_{s}(m)$ for some power sum $\zeta_{s} \in \mathbb{Q} \Sigma$ integer-valued on the progression $A_{s}$.

Choose a progression, say $A_{0}$, that contains infinitely many elements $m \in A$. For all $m \in A \cap A_{0}$ large enough,

$$
a_{0}(m)=\lfloor\sqrt{\alpha(2 m+r)}\rfloor \in\left\{\zeta_{0}(m)-1, \zeta_{0}(m), \zeta_{0}(m)+1\right\} .
$$

We claim that for all large enough $m \in A_{0}$, either $a_{0}(m)=\zeta_{0}(m)-1$, or $a_{0}(m)=\zeta_{0}(m)$, or $a_{0}(m)=\zeta_{0}(m)+1$. In fact, $a_{0}(m)=\zeta_{0}(m)-1$ when both $\alpha(2 m+r)-\zeta_{0}(m)^{2}+2 \zeta_{0}(m)-1 \geq 0$ and $\alpha(2 m+r)-\zeta_{0}(m)^{2}<0 ; a_{0}(m)=$ $\zeta_{0}(m)$ when both $\alpha(2 m+r)-\zeta_{0}(m)^{2} \geq 0$ and $\alpha(2 m+r)-\zeta_{0}(m)^{2}-2 \zeta_{0}(m)-1$ $<0$; and $a_{0}(m)=\zeta_{0}(m)+1$ when both $\alpha(2 m+r)-\zeta_{0}(m)^{2}-2 \zeta_{0}(m)-1 \geq 0$ and $\alpha(2 m+r)-\zeta_{0}(m)^{2}-4 \zeta_{0}(m)-4<0$. Since $\alpha$ and $\zeta_{0}$ are power sums, each of the above pairs of inequalities can hold for all large $m \in \mathbb{N}$ or for just finitely many $n \in \mathbb{N}$. Since (27) holds for infinitely many $n \in \mathbb{N}$, we have proved that for all large enough $m \in A_{0}, a_{0}(m)=\beta_{0}(m)$ for some power sum $\beta_{0} \in \mathbb{Q} \Sigma$ integer-valued on the progression $A_{0}$ (recall that $\zeta_{0}-1, \zeta_{0}+1 \in \mathbb{Q} \Sigma$ are integer-valued on $A_{0}$ ).

If $R=0$, the proof is complete. Note that since $\alpha$ was supposed to be nonconstant, also $a_{0}(m)$ is a nonconstant power sum on the progression $A_{0}$.

Suppose now $R>0$, and suppose by contradiction that for some arithmetic progression $\mathcal{P} \subseteq A_{0}$ that contains infinitely many elements of $A$, there exists $h \in \mathbb{N}, 1 \leq h \leq R$, such that for all large enough $m \in \mathcal{P}$ and every $i=0, \ldots, h-1, a_{i}(m)=\beta_{i}(m)$ for some power sum $\beta_{i} \in \mathbb{Q} \Sigma$ integer-valued on $\mathcal{P}$, but there does not exist an arithmetic progression $\mathcal{P}^{\prime} \subseteq \mathcal{P}$ and a power sum $\beta_{h} \in \mathbb{Q} \Sigma$ integer-valued on $\mathcal{P}^{\prime}$ such that $a_{h}(m)=\beta_{h}(m)$ for all large enough $m \in \mathcal{P}^{\prime}$.

We can exclude the case $h=R$, since for $m \in A \cap \mathcal{P}$ large enough we have $a_{R}(m)=2 a_{0}(m)=2 \beta_{0}(m) \in \mathbb{Q} \Sigma$, and $\beta_{0}$ is integer-valued on $\mathcal{P}$. Put $a(m):=\left[a_{0}(m) ; a_{1}(m), \ldots, a_{h-1}(m)\right]=p_{h-1}(m) / q_{h-1}(m) \in \mathbb{Q}$. Since $a_{i}(m) \in \mathbb{Q} \Sigma$ for every $i=0, \ldots, h-1$, we have

$$
|\sqrt{\alpha(2 m+r)}-a(m)|^{-1}=\frac{\sqrt{\gamma(m)}+\tau(m)}{\xi(m)}=: \alpha_{h}(m)
$$

for every large enough $m \in A \cap \mathcal{P}$ and for certain power sums $\gamma, \tau$ and $\xi \in \mathbb{Q} \Sigma, \xi$ not identically zero. 
We claim that for every $\varepsilon>0$ there is no power $\operatorname{sum} \zeta \in \mathbb{Q} \Sigma$ such that (29)

$$
\left|\alpha_{h}(m)-\zeta(m)\right| \ll e^{-m \varepsilon} .
$$

In fact, if such a power sum existed, in view of (29) we would have

$$
\left|\alpha_{h}(m)-\zeta(m)\right|<1
$$

for all large enough $m \in A \cap \mathcal{P}$, which implies that for all $m \in A \cap \mathcal{P}$ large enough,

$$
a_{h}(m)=\left\lfloor\alpha_{h}(m)\right\rfloor \in\{\lfloor\zeta(m)\rfloor-1,\lfloor\zeta(m)\rfloor,\lfloor\zeta(m)\rfloor+1\} .
$$

But since $\zeta$ has integral roots and rational coefficients, there would exist an arithmetic progression $\mathcal{P}^{\prime} \subseteq \mathcal{P}$, containing infinitely many elements of $A$, such that for all large enough $m \in A \cap \mathcal{P}^{\prime},\lfloor\zeta(m)\rfloor=\zeta^{\prime}(m)$ for some power sum $\zeta^{\prime} \in \mathbb{Q} \Sigma$ integer-valued on $\mathcal{P}^{\prime}$. This would entail (by the same argument as after formula (27)) that for $m \in \mathcal{P}^{\prime}$ large enough, $a_{h}(m)=\left\lfloor\alpha_{h}(m)\right\rfloor=$ $\beta(m)$ for a power sum $\beta \in \mathbb{Q} \Sigma$ integer-valued on $\mathcal{P}^{\prime}$, a contradiction proving that $\alpha_{h}$ satisfies the assumption of Theorem 3.1.

By the definition of $\alpha_{h}(m)$, the length of the period of its continued fraction is $R$ again, when $m \in A \cap \mathcal{P}$. Let

$$
\alpha_{h}(m)=\left[a_{0}^{\prime}(m) ; \overline{a_{1}^{\prime}(m), \ldots, a_{R}^{\prime}(m)}\right]
$$

and let $p_{i}^{\prime}(m) / q_{i}^{\prime}(m), i=0,1, \ldots$, with $q_{0}^{\prime}(m)=1$, be the sequence of its convergents. We have $a_{i}^{\prime}(m)=a_{i+h}(m)$ for $i+h \leq R, a_{i}^{\prime}(m)=a_{i+h-R}(m)$ for $i+h>R$, and

$$
a_{i+1}^{\prime}(m)<\left|\alpha_{h}(m)-\frac{p_{i}^{\prime}(m)}{q_{i}^{\prime}(m)}\right|^{-1} \quad \text { for } i \geq 0 .
$$

Since $\alpha_{h}$ satisfies the assumption of Theorem 3.1, for some $\varepsilon>0$ to be fixed later there exist $k \geq 3$ and $Q>1$ as in that statement. As in the proof of Theorem 3.1, we can put $Q:=e^{\varepsilon}$.

As in the proof of Corollary 3.3, we have again the inequality $q_{i}^{\prime}(m)<$ $Q^{2 m+r}$ for every $i=0, \ldots, R$ and $m \in A \cap \mathcal{P}$ large enough, i.e. we can apply Theorem 3.1 to $\alpha_{h}(m)$ with $p=p_{i}^{\prime}(m), q=q_{i}^{\prime}(m)$ and some $\varepsilon>0$ to be fixed later. We infer that for every $i \geq 0$ and for $m \in A \cap \mathcal{P}$ large enough,

$$
\left|\alpha_{h}(m)-\frac{p_{i}^{\prime}(m)}{q_{i}^{\prime}(m)}\right| \geq q_{i}^{\prime}(m)^{-k} e^{-m \varepsilon} .
$$

Recalling that $0<q_{i}^{\prime}(m)<Q^{2 m+r}=e^{(2 m+r) \varepsilon}$ for every $i=0, \ldots, R$ and $m \in A \cap \mathcal{P}$ large enough, and considering the inequality (31) for $i=R-h-1$, together with (30), we get, for large enough $m \in A \cap \mathcal{P}$,

$$
\begin{aligned}
a_{R}(m)=a_{R-h}^{\prime}(m) & \leq\left|\alpha_{h}(m)-\frac{p_{R-h-1}^{\prime}(m)}{q_{R-h-1}^{\prime}(m)}\right|^{-1} \leq q_{R-h-1}^{\prime}(m)^{k} e^{m \varepsilon} \\
& <Q^{(2 m+r) k} e^{m \varepsilon}=e^{\varepsilon((2 m+r) k+m)}<e^{m \varepsilon^{\prime}}
\end{aligned}
$$


for $\varepsilon^{\prime}=3 k \varepsilon$. Choosing $\varepsilon<(\ln 2) / 3 k$ (i.e. $\varepsilon^{\prime}<\ln 2$ ), we see that for $m \in A \cap \mathcal{P}$ large enough,

$$
a_{R}(m) \ll 2^{m(1-\delta)} \quad \text { for some } \delta>0 .
$$

Recalling that $a_{0}(m) \in \mathbb{Q} \Sigma$ is nonconstant for $m \in A \cap \mathcal{P}$, from the relation

$$
a_{R}(m)=2 a_{0}(m) \gg 2^{m}
$$

we get a contradiction, proving that there exists an arithmetic progression $\mathcal{P}$ such that (5) holds for all but finitely many $m \in \mathcal{P}$.

We now prove the functional relation. From (5) we get

$$
\sqrt{\alpha(2 m+r)}=\beta_{0}(m)+\frac{1}{\tau(m)} \quad \text { for } m \in \mathcal{P},
$$

where $\tau(m)$ has the continued fraction expansion

$$
\tau(m)=\left[\overline{\beta_{1}(m), \ldots, \beta_{R}(m)}\right] .
$$

This means that for $m \in \mathcal{P}$,

$$
\tau(m)=\left[\beta_{1}(m), \ldots, \beta_{R}(m), \tau(m)\right],
$$

which can be rewritten as a quadratic equation

$$
q_{R}^{\prime \prime}(m) \tau(m)^{2}+\left(q_{R-1}^{\prime \prime}(m)-p_{R}^{\prime \prime}(m)\right) \tau(m)-p_{R-1}^{\prime \prime}(m)=0,
$$

where $p_{i}^{\prime \prime}(m) / q_{i}^{\prime \prime}(m)=\left[\beta_{1}(m), \ldots, \beta_{i}(m)\right]$.

It is well known that $p_{i}^{\prime \prime}(m), q_{i}^{\prime \prime}(m)$ satisfy the recursive equations $p_{i+2}^{\prime \prime}(m)$ $=\beta_{i+2}(m) p_{i+1}^{\prime \prime}(m)+p_{i}^{\prime \prime}(m)$ and $q_{i+2}^{\prime \prime}(m)=\beta_{i+2}(m) q_{i+1}^{\prime \prime}(m)+q_{i}^{\prime \prime}(m)$ for all $i \geq-1$, with initial values $p_{0}^{\prime \prime}(m)=q_{-1}^{\prime \prime}(m)=1$ and $q_{0}^{\prime \prime}(m)=p_{-1}^{\prime \prime}(m)=0$. It follows that $p_{R-1}^{\prime \prime}(m), p_{R}^{\prime \prime}(m), q_{R-1}^{\prime \prime}(m)$ and $q_{R}^{\prime \prime}(m)$ appearing in $(34)$ are all power sums in $\mathbb{Q} \Sigma$. This means that the equation (34) either holds for just finitely many $m \in \mathbb{N}$, or holds identically. Since it holds for all $m \in \mathcal{P}$, i.e. for infinitely many $m$, it must hold identically, concluding the proof.

Acknowledgements. The author is grateful to Prof. U. Zannier for bringing this problem to his attention and to Prof. P. Corvaja for valuable comments and remarks.

\section{References}

[1] Y. Bugeaud and F. Luca, On the period of the continued fraction expansion of $\sqrt{2^{2 n+1}+1}$, Indag. Math. 16 (2005), 21-35.

[2] P. Corvaja and U. Zannier, Diophantine equations with power sums and universal Hilbert sets, ibid. 9 (1998), 317-332.

[3] - - - On the length of the continued fraction for the values of quotients of power sums, J. Théor. Nombres Bordeaux 17 (2005), 737-748.

[4] L. K. Hua, On the least solution to Pell's equation, Bull. Amer. Math. Soc. 48 (1942), 731-735.

[5] A. Ya. Khinchin, Continued Fractions, Dover, 1997. 
[6] H. W. Lenstra, Jr., Solving the Pell equation, Notices Amer. Math. Soc. (2) 49 (2002), 182-192.

[7] A. Schinzel, On some problems of the arithmetical theory of continued fractions, Acta Arith. 6 (1961), 393-413.

[8] -, On some problems of the arithmetical theory of continued fractions II, ibid. 7 (1962), 287-298.

[9] W. M. Schmidt, Diophantine Approximation, Lecture Notes in Math. 785, Springer, 1980.

[10] —, Diophantine Approximations and Diophantine Equations, Lecture Notes in Math. 1467, Springer, 1991.

Via San Rocco 130

I-33100 Udine, Italy

E-mail: scremin@finanz.math.tu-graz.ac.at

Received on 31.5.2004

and in revised form on 6.9.2005 